LETTER TO THE EDITOR

\title{
Extracting quantum dynamics from genetic learning algorithms through principal control analysis
}

\author{
J L White, B J Pearson and P H Bucksbaum \\ FOCUS Center, Physics Department, University of Michigan, Ann Arbor, MI, 48109-1120, USA \\ E-mail: phb@umich.edu
}

Received 1 October 2004

Published 6 December 2004

Online at stacks.iop.org/JPhysB/37/L399

doi:10.1088/0953-4075/37/24/L02

\begin{abstract}
Genetic learning algorithms are widely used to control ultrafast optical pulse shapes for photo-induced quantum control of atoms and molecules. An unresolved issue is how to use the solutions found by these algorithms to learn about the system's quantum dynamics. We propose a simple method based on covariance analysis of the control space, which can reveal the degrees of freedom in the effective control Hamiltonian. We have applied this technique to stimulated Raman scattering in liquid methanol. A simple model of two-mode stimulated Raman scattering is consistent with the results.
\end{abstract}

(Some figures in this article are in colour only in the electronic version)

The central challenge of coherent control of quantum dynamics is to find the optimal path to guide a quantum system from its initial state to some target final state $[1,2]$. Several theoretical methods have been developed to aid this search [3, 4], and there has been considerable experimental success as well [5-7]. However, in all but the simplest systems, the search is hampered by incomplete knowledge of the system Hamiltonian. Strongly coupled systems such as large molecules in condensed phase are so complicated that it is nearly impossible to calculate optimal pulse shapes in advance.

Feedback learning algorithms overcome this limitation by using the physical system itself to explore its own quantum dynamics through an experimental search [8]. A typical search experiment compares the ability of several thousand different shaped laser pulses to transform the system $|\psi\rangle$ from its initial state at time $t=0$ to some desired target state $|\chi\rangle$ at a later target time $t=T$. Examples of transformations that have been studied include molecular photodissociation, atomic photoexcitation and photoionization. The pulse shapes are selected through a fitness-directed search protocol, such as a genetic algorithm [9]. The fitness is a 
measured quantity proportional to the objective functional $J\left[H ; x_{i}\right]$, which is the square of the projection of $|\chi\rangle$ onto $|\psi\rangle$ at the end of the experiment:

$$
J\left[H ; x_{i}\right]=|\langle\chi(T) \mid \psi(T)\rangle|^{2} .
$$

$J$ depends on the Hamiltonian $H$ for the system evolution, which depends in turn on the laser electric field $E(t)$ determined by the settings of the $n$ pulse shape control parameters $x_{i}$, $i=1, \ldots, n . J$ reaches its extreme value for the optimal pulse. This pulse can be calculated using optimal control theory if $H$ is known $[4,10]$; otherwise, it must be discovered through the learning search.

Several recent papers have suggested modifications or extensions of learning feedback that can measure properties of the system Hamiltonian [11-15]. Here we propose a different approach based on the analysis of the trial experiments. We will show that the ensemble of trial pulse shapes can reveal essential features of the dynamics.

Genetic algorithms and similar evolutionary search strategies have many different variations [16]. Our implementation starts with approximately 50 randomly generated optical pulse shapes produced by spectrally filtering an ultrafast laser pulse [17]. Each pulse shape is described by a column matrix of control parameters $x_{i}$ called a genome consisting of about 25 numbers (genes), each encoding the amplitude and/or phase of a different segment of the optical spectrum. The control target is measured for each pulse shape. Then the algorithm creates a new generation of pulse shapes by combining attributes of the fittest members of the previous generation [18]. After several generations, the pulse shapes usually cluster near high fitness regions of the search space. When the algorithm finds a pulse shape or several shapes that cannot be improved over many generations, the search stops, and the highest fitness pulse shape is declared the optimal solution to the search. We test 1000 to 10000 pulse shapes in a typical experiment. We maintain a record of every pulse shape, its fitness and its parentage (genealogy).

The learning algorithm achieves control without prior knowledge of the system Hamiltonian, and has far more degrees of freedom $n$ than the minimum required for control. The number of possible solutions is exponential in $n$. In a typical search, the phase of each colour is adjusted by the spectral phase filter to a precision of about $10^{\circ}$, so there are $2^{5}$ possible values of each gene. This means that the number of possible solutions for a genome of length 25 is $2^{5 \times 25} \simeq 4 \times 10^{37}$. Genetic algorithms can search this large state space with great efficiency [9]. Unfortunately, simply finding a good solution has not often provided significant insight into the system dynamics or Hamiltonian. The optimal pulse shape found by the learning algorithm, while sufficient to achieve control, is often complicated and may contain unnecessary features.

The conditions for reaching an extremum in $J\left[H ; x_{i}\right]$ may only depend on two or three essential features of the control field $E(t)$. These features are not obvious in the successful genome because they may depend on all 25 genes. The Hamiltonian could be written in a much simpler form if these essential degrees of freedom $\left(u_{j}\right)$ were found. Here we show how to establish the $u_{j}$ through covariance analysis of the pulse shapes evaluated during the learning search [19]. Covariance analysis is commonly used to reduce the dimensionality of and to find patterns in high-dimensional data sets.

We propose to apply these techniques, not to sets of data, but to the control space for the experiment. Linear combinations of genes with high fitness should appear correlated in the fitness-driven genetic algorithm. These correlated linear combinations correspond to the principal components of the control field that direct the quantum dynamics under investigation. Principal control analysis is the application of covariance techniques to a fitness-directed search. 
Principal control analysis is implemented on our system by calculating the covariance matrix of the set of all pulse shapes in the search, defined by

$$
C_{i j}=\left\langle\delta_{i} \delta_{j}\right\rangle-\left\langle\delta_{i}\right\rangle\left\langle\delta_{j}\right\rangle,
$$

where the expressions $\delta_{i}=x_{i+1}-x_{i}, i=1, \ldots, n-1$ are the nearest-neighbour phase differences. By using the phase differences in the analysis we remove the ambiguity associated with the unimportant global phase. The covariance matrix is not the only measure of correlation. We could also weight the terms of the covariance matrix using the fitness, or normalize each term to the individual gene variance. In this paper, we will use the simple covariance. This is appropriate because all the $\delta_{i}$ are of the same type.

Once the covariance matrix is determined, we calculate its eigenvectors and eigenvalues. Each eigenvalue $\lambda_{j}$ is the variance of the projections onto the corresponding eigenvector. This has a special meaning for a learning control search: it shows how far the control setting moved during the learning process. A small subset of eigenvalues usually contains most of the weight of the trace of the covariance matrix. The correlations of the projections with the pulse shape fitnesses allow us to determine which control directions were most important for the physical process under consideration. The controls expressed in the basis of the eigenvectors are uncorrelated: each of these controls changes the fitness without correlation with the others over the search set.

We propose that these eigenvectors with the largest fitness correlation are the essential control directions $\left(u_{j}\right)$. We expect the eigenvectors with the larger eigenvalues to be most strongly correlated with the fitness of a pulse shape solution. Conversely, a low correlation indicates those eigenvectors that have not contributed substantially to increasing the fitness during the search. These eigenvectors correspond to extraneous dimensions, which could be eliminated (i.e., their projection set to zero) without losing substantial control.

By projecting the GA solutions onto the $k<n$ eigenvectors that correlate best with the fitness (the principal controls), we reduce the dimension of the control space. The solutions with highest fitness, when expressed in the reduced basis of the principal controls, represent the essential features of the search solutions. The objective functional also takes on a simpler form in this basis:

$$
J=J\left[H ; u_{1, \ldots, k}\right]
$$

where each $u_{j}$ can assume a range of values on the order of $\pm \sqrt{\lambda_{j}}$. The search for the specific target state is now a matter of optimizing each control $u_{j}$ over this range.

In summary, we propose to apply covariance techniques to the control space of learning feedback experiments. Control values derived from the genomes are analysed by a covariance matrix, defined in equation (2). The matrix eigenvectors are independent control directions. The correlation of the fitness with the eigenvectors suggests which control directions are the most important. The corresponding eigenvalues indicate the necessary excursion along each axis. Therefore, searches conducted in the eigenvector basis are more efficient. Finally, the essential features of the search solutions are found by projecting the optimal learning control solutions onto the important control directions.

We now apply this analysis to a well-studied control problem: the selective excitation of vibrational modes in liquid methanol. The experiment has been described previously $[18,20,21]$. An intense shaped $800 \mathrm{~nm}$ ultrafast laser pulse (the pump laser) is focused into a cell containing methanol. Above a threshold fluence, the pump induces stimulated Raman scattering into either the symmetric or antisymmetric Raman-active $\mathrm{C}-\mathrm{H}$ stretch mode. Either mode can be selectively excited by adjusting the shape of the pump pulse through phase shaping and/or amplitude shaping of its spectrum. These experiments led to the demonstration of a 


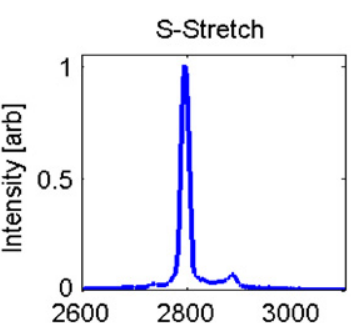

(a)
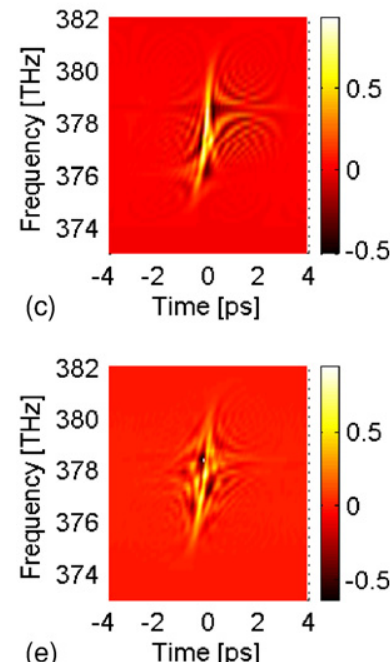

(e)

Figure 1. Left: Raman spectra (a) and Wigner representation (c) of the optimal pulse shape after optimizing the symmetric $\mathrm{C}-\mathrm{H}$ stretch mode. Wigner representation (e) of the control pulse shape found by principal control analysis. Right: similar for antisymmetric stretch.

control mechanism for SRS in methanol based on periodicity in $I(t)$ [20]. In this paper, we show that PCA emphasizes these same features of the control field.

Figure 1 depicts the results of a phase-only feedback control experiment. Panels (a) and (b) show the Raman spectra produced by the optimal pulse for the symmetric stretch and antisymmetric stretch, respectively. The two searches evaluated a total of 3240 pulse shapes. Moderate fitness increases were observed for either target mode after 25 generations. The fitness increase was greater for the symmetric mode. This is typical of our searches based on phase-only control [21].

A Wigner representation of the pulse shape solution found by the learning algorithm is plotted in panels (c) and (d). The Wigner function is a spectrally resolved field auto-correlation:

$$
W(\omega, t)=\int \mathrm{d} \omega^{\prime} E\left(\omega-\omega^{\prime}\right) E^{*}\left(\omega+\omega^{\prime}\right) \mathrm{e}^{-2 \mathrm{i} \omega^{\prime} t} .
$$

Wigner representations are complete time-frequency spectrograms of the optical control field (up to a global phase), but the important features leading to control of the methanol are obscure. The inability to interpret the result easily is typical of many GA search solutions [22, 23].

The principal control analysis of this problem begins with a single covariance matrix (equation (2)) for the entire collection of pulse shapes evaluated in the two feedback experiments. The physical system under control was the same in the two problems; only the target was different. We therefore expect the independent searches to be nearly spanned 

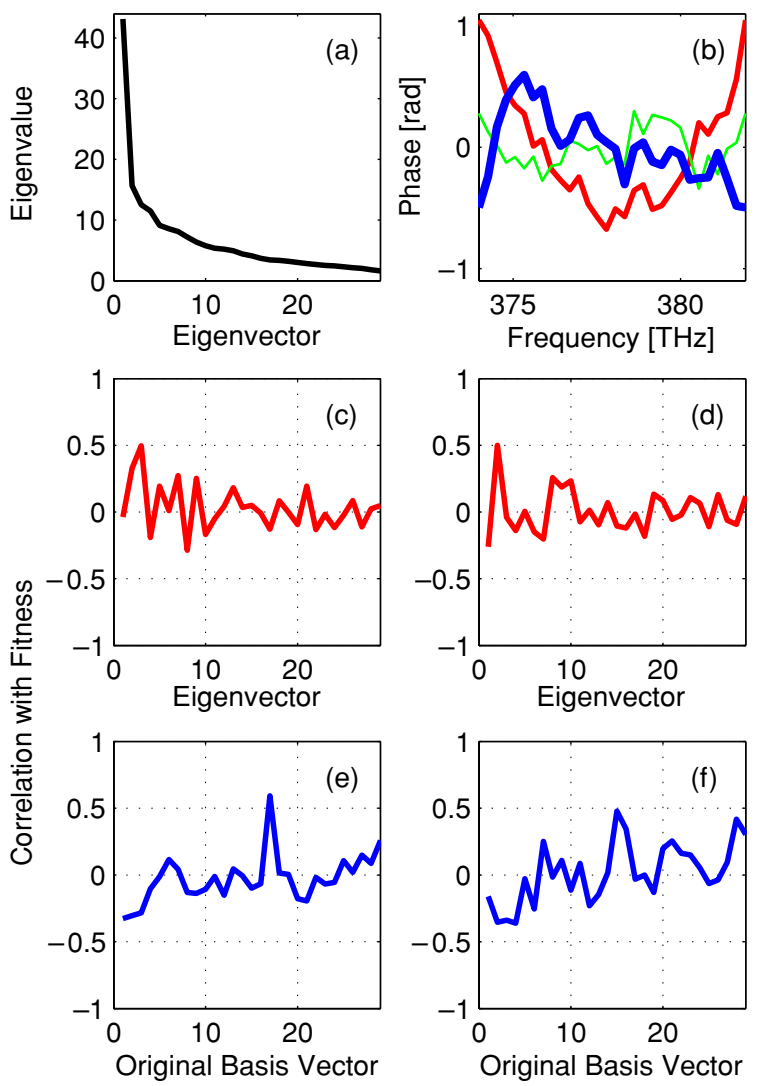

Figure 2. Panel (a): eigenvalues of the covariance matrix in descending order. Panel (b): phase functions associated with the three principal control eigenvectors. For the symmetric stretch, the fitness correlates well with the third (thin) and second (middle) eigenvectors. Antisymmetric stretch fitness correlates well with the second (middle) and first (thick) eigenvectors. Panel (c): correlation of fitness $\mathrm{f}$ with the control vectors for the symmetric stretch ordered by eigenvalue. Specifically, the correlation is $\left(\left\langle\eta_{i} f\right\rangle-\left\langle\eta_{i}\right\rangle\langle f\rangle\right) / \sigma_{\eta_{i}} \sigma_{f}$. Panel (d): similar for the antisymmetric stretch. Panel (e): the correlation in the original basis ordered by frequency: $\left(\left\langle\delta_{i} f\right\rangle-\left\langle\delta_{i}\right\rangle\langle f\rangle\right) / \sigma_{\delta_{i}} \sigma_{f}$ for the symmetric stretch. Panel (f): similar for the antisymmetric stretch.

by a small number of eigenvector controls. The covariance matrix is not simple to interpret because the principal controls in this problem are widely distributed among all of the control settings in the search space. However, the essential features of the control problem begin to emerge if the covariance matrix is diagonalized. This can be seen in figure 2(a), where the eigenvalues of the covariance matrix are plotted in descending order. The algorithm has made large excursions only along the few control directions that have large eigenvalues. The phase functions associated with the three largest eigenvalues are shown in figure 2(b). The correlation of eigenvector with fitness is plotted in figure 2(c) for the symmetric stretch and in figure 2(d) for the antisymmetric stretch. We find that eigenvectors corresponding to the three largest eigenvalues are also the three control directions that correlate most strongly with fitness. Therefore, we propose that the dimension of the search space can be reduced without inhibiting control. We take these three eigenvectors to be the principal controls.

Each pulse shape can now be re-expressed in the eigenvector basis. To arrive at the essential features of the best pulse, we calculate the projections $\eta_{k}$ of the optimal pulse 

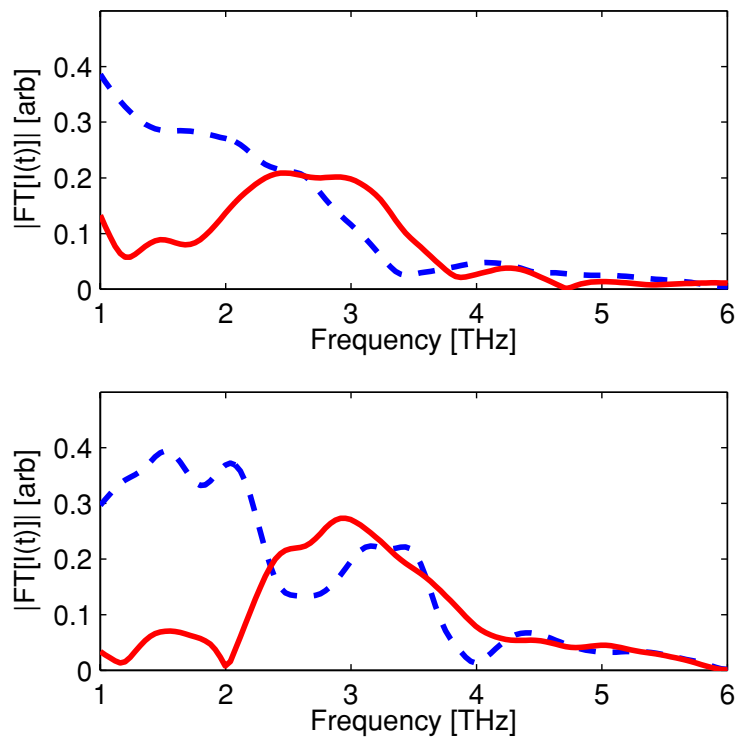

Figure 3. Top: magnitude of the Fourier transform of $I(t)$ for the optimal pulse shape found by the learning algorithm (dashed) and for the essential pulse shape found through principal control analysis (solid) for the symmetric stretch mode. Bottom: similar for the antisymmetric stretch mode.

shape onto the principal control directions $u_{k}$. When these $u_{k}$ are expressed in the original basis, their components are individual discrete frequencies that make up the field. Therefore, $\sum_{j=1}^{k} \eta_{j} u_{j}(\omega)$ produces a pulse that contains traits necessary to achieve the target, with minimal extraneous features. Figures 1(e) and (f) show the Wigner plot of this essential pulse for the symmetric stretch and antisymmetric stretch, respectively. The essential pulse for the symmetric stretch preserves $66 \%$ of the original pulse shape vector $\left(\sum_{j=1}^{3} \eta_{j}^{2}(\omega)=66 \%\right)$. For the antisymmetric stretch, this number is $86 \%$.

Our analysis so far has been independent of the specific nature of the physical system or the dynamical Hamiltonian; however, the principal control directions contain features that will be found in the dynamical Hamiltonian since the laser electric field now only depends on a few parameters:

$$
H(t)=H\left(E\left[u_{j=1, \ldots, k} ; t\right]\right) .
$$

This dependence can provide important constraints. We find that the results of PCA are consistent with the control mechanism previously demonstrated [20]. The control mechanism is based on periodicity in $I(t)$, with the Fourier transform of $I(t)$ revealing the most important Raman coupling frequencies in the problem [24]. Figure 3 (top) shows the magnitude of the $F T[I(t)]$ for the optimal pulse shape found by the learning algorithm for the symmetric stretch mode (dashed) and for the same pulse shape projected onto the principal control directions $u_{k}$ (solid). Figure 3 (bottom) shows similar plots for the antisymmetric mode.

For both modes, projecting the optimal pulses onto the principal control directions enhances the frequency components around $3 \mathrm{THz}$. The coupling frequency found through principal control analysis agrees with the model based on the mode separation in methanol. Additionally, comparing the phases of the Fourier transforms for each mode yields a phase difference of $\pi / 2$ in the region around $3 \mathrm{THz}$, consistent with the model described in [20]. 
In conclusion, we have shown how covariance analysis of a genetic search algorithm can uncover essential features of the dynamical Hamiltonian. Although our example involved only phase shaping of the optical field, this technique should be applicable to any system where fitness-directed learning algorithms have been used to reveal the path from an initial quantum state to a target. Principal control analysis can also be incorporated into the experimental search protocol. By discovering the principal controls, it should be possible to search the space more efficiently, and to test ideas about the system dynamics as the search is proceeding. The method should be most useful in cases where the dynamics can be described by only a few principal degrees of freedom, which are linear combinations of the control parameters of the search space.

\section{Acknowledgments}

We thank Anna Amirdjanova, Jayson Cohen, Vladimir Dergachev, Chitra Rangan, Ben Recht and Tom Weinacht for valuable discussions. This work was supported by the National Science Foundation under grant 9987916.

\section{References}

[1] Tannor D J and Rice S A 1988 Adv. Chem. Phys. 70441

[2] Brumer P and Shapiro M 1992 Ann. Rev. Phys. Chem. 43257

[3] Tannor D J, Kosloff R and Rice S A 1986 J. Chem. Phys. 855805

[4] Peirce A P, Dahleh M A and Rabitz H 1988 Phys. Rev. A 374950

[5] Shnitman A, Sofer I, Golub I, Yogev A, Shapiro M, Chen A and Brumer P 1996 Phys. Rev. Lett. 762886

[6] Gordon R J 1995 Science 27077

[7] Assion A, Baumert T, Bergt M, Brixner T, Kiefer B, Seyfried V, Strehle M and Gerber G 1998 Science 282919

[8] Judson R S and Rabitz H 1992 Phys. Rev. Lett. 681500

[9] Holland J H 1992 Sci. Am. 26766

[10] Hornung T, Motzkus M and Vivie-Riedle R 2002 Phys. Rev. A 65 021403(R)

[11] Hornung T, Meier R and Motzkus M 2000 Chem. Phys. Lett. 326445

[12] Geremia J M, Weiss E H and Rabitz 2001 Chem. Phys. 267209

[13] Geremia J M and Rabitz H 2002 Phys. Rev. Lett. 89263902

[14] Mitra A and Rabitz H 2003 Phys. Rev. A 6733407

[15] Daniel C, Full J, González L, Lupulescu C, Manz J, Rerli A, Vajda Š and Wöste L 2003 Science 299536

[16] Davis L 1991 Handbook of Genetic Algorithms (New York: Van Norstrand Reinhold)

[17] Tull J X, Dugan M A and Warren W S 1990 Adv. Magn. Opt.Reson. 201

[18] Pearson B J, White J L, Weinacht T C and Bucksbaum P H 2001 Phys. Rev. A 63063412

[19] Jolliffe I T 2002 Principal Component Analysis 2nd edn (Berlin: Springer)

[20] Pearson B J and Bucksbaum P H 2004 Phys. Rev. Lett. 92243003

[21] Pearson B J, Morris D S, Weinacht T C and Bucksbaum P H 2002 Femtochemistry and Femtobiology: Ultrafast Dynamics in Molecular Science ed A Douhal and J Santamaria (Singapore: World Scientific) p 399

[22] Levis R J, Menkir G M and Rabitz H 2001 Science 292709

[23] Brixner T, Damrauer N H, Niklaus P and Gerber G 2001 Nature 41457

[24] Meshulach D and Silberberg Y 1998 Nature 396239 